\title{
Voix de « ceux qui ne sont rien » en Asie du Sud-Est
}

Alexis Michaud, Minh-Châu Nguyễn et Likun He 和丽昆

\section{OpenEdition}

\section{Journals}

Édition électronique

URL : https://journals.openedition.org/clo/7019

DOI : $10.4000 /$ clo. 7019

ISSN : 2266-1816

Éditeur

INALCO

\section{Édition imprimée}

Date de publication : 13 août 2020

Pagination : 147-154

ISBN : 9782858313501

ISSN : 0396-891X

\section{Référence électronique}

Alexis Michaud, Minh-Châu Nguyễn et Likun He 和丽昆, «Voix de « ceux qui ne sont rien » en Asie du Sud-Est », Cahiers de littérature orale [En ligne], Hors-Série | 2020, mis en ligne le 02 septembre 2020, consulté le 23 mars 2022. URL : http://journals.openedition.org/clo/7019 ; DOI : https://doi.org/ $10.4000 /$ clo.7019

\section{(c) $(1)(9$}

Cahiers de littérature orale est mis à disposition selon les termes de la Licence Creative Commons Attribution - Pas d'Utilisation Commerciale 4.0 International. 


\title{
Voix de « ceux qui ne sont rien $\gg$ en Asie du Sud-Est
}

\author{
Alexis Michaud \\ LACITO - CNRS - Sorbonne Nouvelle - Inalco \\ Minh-Châu NGUYỄN \\ LACITO - CNRS - Sorbonne Nouvelle - Inalco \\ Likun HE 和丽昆 \\ Yunnan Minzu University, Chine
}

L'équipe éditoriale des Cahiers de littérature orale invitant des contributions autour des « oralités contestataires », nous proposons un aperçu de trois documents de littérature orale provenant de civilisations d'Asie Orientale et du Sud-Est : une historiette naxi, un récit khamou, et une chanson vietnamienne, chacun en dialogue avec un texte (article ou livre) tiré de l'actualité sociale et politique.

\section{Robin des bois}

Le titre de l'article de Mediapart « Emmanuel Macron reste un "Robin des Bois à l'envers" ${ }^{1} \gg$ se passe d'explication, tant la figure de Robin des Bois, qui détroussait les riches au profit des pauvres, est bien établie dans la culture populaire

1. https://www.mediapart.fr/journal/france/050220/emmanuel-macron-reste-unrobin-des-bois-l-envers (5 février 2020). 
européenne. Les cultures d'Asie du Sud-Est ont, elles aussi, leurs héros qui, dans l'espace du conte, vengent les petites gens de l'oppression. Chez les Naxi (Yunnan, Chine), dirigés d'une poigne de fer (du XIII ${ }^{\mathrm{e}}$ au XVIII ${ }^{\mathrm{e}}$ siècle) par une dynastie de seigneurs qui enrégimentaient la société (faisant des hommes des soldats et des femmes des hommes en civil $^{2}$ ), les histoires favorites de la tradition orale restent, jusqu'à aujourd'hui, celles de l'espiègle A-i-nder. Le tyran devenu gâteux dans ses vieux jours, son malicieux serviteur A-i-nder le manœuvre à sa guise, d'un épisode rocambolesque à l'autre.

Un jour, en préparant la pâtée pour les porcs, A-i-nder y a mis tout un seau de poivre du Sichuan. Puis il a crié : «Monseigneur, monseigneur ! Nos porcs sont malades, ils sont pris de convulsions ! » Les porcs, avec tout le poivre qu'il leur avait fait manger, ils avaient l'écume aux babines! Alors, le vieux a dit : «Hou là là, il faut les abattre et les vendre tout de suite ! » A-i-nder les a abattus, et il est parti vendre la viande par les rues de la ville. «Achetez ma viande de cochon malade ! Achetez ma viande de cochon malade ! » Et bien sûr, il n'a pas trouvé acquéreur. De retour, il a dit : «Ah là là, ça ne s'est pas du tout vendu ! » Le vieux lui a demandé ce qui s'était passé. « Ben j'ai dit "achetez ma viande de cochon malade !” », a-t-il répondu. « Ah, mais ! Demain, tu y retournes, et tu ne dis pas qu'ils étaient malades, enfin ! » lui répond le vieux. Le lendemain, A-i-nder a changé de discours : «Achetez ma viande de cochons pas malades du tout ! » Il obtient bien sûr le même effet que la veille. Le troisième jour [la viande n'étant plus assez fraîche pour être vendue] A-i-nder a tout distribué aux familles pauvres. Il était comme ça, A-i-nder : il bernait son monde, mais il avait bon cœur ; c'était le défenseur des pauvres, et c'est pour leur bénéfice qu'il faisait les quatre cent coups chez le vieux seigneur. ${ }^{3}$

Le conte facétieux renverse la domination qu'exerçait le seigneur redouté. On peut consulter les savoureuses histoires d'A-i-nder, et de ses cousins plus ou moins lointains, fripons et héros populaires d'autres peuples des contreforts

2. Mathieu, 2003.

3. Pour consulter le document : https://doi.org/10.24397/pangloss-0004712. 
de l'Himalaya, Pumi ${ }^{4}$ ou Khroskyabs ${ }^{5}$, dans la collection Pangloss, archive ouverte (en libre accès) de langues menacées ${ }^{6}$. Les sociétés au sein desquelles se transmettaient ces traditions subissent des transformations rapides, au fil des dégradations environnementales et des orientations fixées par l'État-nation. Les communs numériques ${ }^{7}$ tels que la collection Pangloss offrent un fragile refuge à ces oralités contestataires.

Les histoires d'A-i-nder le roublard au grand cœur inquiètent suffisamment les tenants de la bienséance sociale pour que des descendants des seigneurs naxi aient tenté de les faire interdire, au début $\mathrm{du} \mathrm{XXI}^{\mathrm{e}}$ siècle, en lançant une procédure judiciaire. De même Robin des Bois avait, dit-on, été censuré au temps du maccarthysme, pour apologie du communisme. Censure sans doute sans objet : les renversements carnavalesques ne mettent guère en danger les puissances établies. Des carnavals comme exutoires populaires (et pour les lettrés, la catharsis des belles-lettres), puis le calme revient. Mais si tel se rêve fripon ou voyou le temps d'un récit ou d'une chanson, pour mieux se résigner à son lot quotidien de mécontentement et d'aliénation, l'oralité révèle aussi des failles plus profondes et introduit à des soubresauts sociaux moins anodins.

\section{La victoire des vaincus}

Le titre de l'ouvrage La Victoire des vaincus, dans lequel Edwy Plenel voudrait reconnaître une victoire symbolique à des contestataires brutalement réprimés ${ }^{8}$, n'est pas sans rappeler le poème épique de Cheuang, « aussi monumental pour l'étude de la civilisation d'Asie du Sud-Est que l'est le Ramayana pour l'Inde ou l'Iliade pour l'Occident ${ }^{9} \gg$. Lorsque les Taï de la période pré-bouddhiste ont migré vers l'Ouest, depuis le Nord du Vietnam, ils ont soumis les populations austroasiatiques plus anciennement établies. Le héros de l'histoire, Cheuang, est un Austroasiatique qui résiste à l'avancée des Taï. Dans la version étudiée par James Chamberlain (un manuscrit laotien du XIX ${ }^{\mathrm{e}}$ siècle), Cheuang est tué, mais

4. « Histoire d'Akhustonpa » : https://doi.org/10.24397/pangloss-0003770.

5. On consultera par exemple «Le voleur et le roi »: https://doi.org/10.24397/ pangloss-0005673.

6. https://pangloss.cnrs.fr/.

7. Voir : https://scinfolex.com/2019/06/25/des-lieux-aux-liens-ce-que-les-communs-fonta-la-propriete/.

8. Plenel, 2019.

9. Chamberlain, 1990. 
par les pouvoirs combinés de Then Lo, maître du Ciel et ancêtre mythique des Taï, et d'un esprit magique, Cheuang renaît comme un esprit. À la tête d'une armée d'esprits, il conquiert l'empyrée entier, avec le consentement d'Indra, roi des dieux et Seigneur du Ciel dans la mythologie védique. «Quelle geste plus hérö̈que, et quelle meilleure revanche, aurait pu connaître un peuple subjugué dans le monde d'en-bas ${ }^{10}$ ? »

Un paradoxe de la version laotienne du mythe de Cheuang est qu'elle est entrée dans la tradition orale du conquérant (de langue taï : thai - siamois - et laotien sont des langues extrêmement proches). Michel Ferlus a recueilli au Laos en 1965 une version du récit de Cheuang (Chuang) chez les Khamou (Khmu), peuple austroasiatique ${ }^{11}$. Cette version plus conservatrice constitue un objet d'étude passionnant au plan historique et ethnologique, aussi bien que linguistique, mais pour le propos du moment, on retiendra surtout que le récit de la chute du héros conservait encore, au $\mathrm{XX}^{\mathrm{e}}$ siècle, un pouvoir d'évocation considérable.

Entre les années 1950 et 1960, un mouvement messianique se propagea chez les Khamou et chez d'autres montagnards. Un messie devait venir prendre la tête du peuple khamou et lui apporter des richesses. [...] Certains Khamou, sûrs de la venue prochaine du libérateur, cessèrent les semailles. Devant le danger, l'administration lao arrêta les propagateurs de cette nouvelle croyance et força à la reprise des travaux des champs.

Ce messie était Chuang ${ }^{12}$.

Cette évocation d'un élan messianique nous fournit une transition opportune pour passer de l'oral... au sadique-anal, diraient peut-être certains psychanalystes (bien renseignés, car ils savent écouter). La troisième étape du voyage sera une chanson populaire vietnamienne qui évoque le face-à-face entre « ceux qui ne sont rien » et une classe dominante arrogante. La chanson dit des rêves de renversements brutaux.

\section{Ibid.}

11. Ferlus, 1979. L'enregistrement audio est accueilli par la collection Pangloss : https://doi.org/10.24397/pangloss-0001986. La saisie de la transcription et son alignement temporel avec l'audio font partie des tâches prévues dans les années qui viennent.

12. Ferlus, 1979, p. 364. 


\section{Crépuscules : la surdité, à nos risques et périls}

Le texte ci-dessous ne nous est connu que par l'écrit, celui du lettré Phạm Quỳnh (Phạm, 1930, p. 74-75).

\section{- Thân cô nhui hoa gạo trên cây, thân tôi như đám cỏ may bên} duiơng. Lay trời cho cả gió rung cây, cho hoa rung xuống cỏ may xỏ vào!

- Thân chị như cánh hoa sen, chúng em nhü bèo nhui bọt chang chen diợc nào.

- Lay trời cho cả muia rào, cho sấm cho chớp, cho bão to gió lớn, cho sen chim xuống bèo trèo lên sen!

- Vous êtes, Mademoiselle, comme une fleur dans la haute ramure du kapokier rouge, tandis que je ne suis qu'une herbe rêche au bord du chemin. Puisse le Ciel envoyer une tempête qui secoue l'arbre, et fasse choir la fleur, qu'elle vienne se ficher sur l'herbe piquante !

- Je suis comme une branche de lotus; et vous les benjamins, vous êtes comme des lentilles d'eau, qui n'avez pas votre place auprès de moi.

- Puisse le Ciel nous livrer averses, tonnerre et éclairs : un typhon qui fera ployer le lotus. Tombé dans l'eau trouble, il sera livré aux assauts des lenticules ${ }^{13}$ !

De cette vision, à laquelle la traduction peine à rendre justice, mesurons la violence. À la morgue et au mépris de la belle demoiselle, répond un ressentiment fulgurant (qui, pour le lecteur français, évoquera peut-être les Fleurs du Mal). Voilà qui rappelle que les dominants qui font la sourde oreille (pour ne pas entendre... la volonté générale, par exemple, ni la voix de la science climatologique) le font à nos risques et périls. Le mépris porte en germe le risque de spirales de violence. $\mathrm{Si}$

13. Traduction de Phạm Quỳnh : «Vous êtes, mademoiselle, comme la fleur du faux-cotonnier qui est sur l'arbre. Je suis, moi, comme une touffe d'herbe piquante qui pousse sur le bord du chemin. Je prie le ciel de faire un grand vent qui secouera l'arbre, fera tomber à terre la fleur pour qu'elle soit piquée par l'herbe !

- Je suis, moi, une branche de lotus, et vous autres, hommes, vous êtes des lentilles d'eau qui ne sauraient pousser parmi les lotus ;

- Je prie le ciel d'amener une averse, avec tonnerre et éclairs, ouragan et typhon, pour que la fleur de lotus soit immergée et que les lentilles montent dessus. » 
le contrat social paraît bafoué, il y a un risque de « donner raison à ces haines et violences que l'on s'est tant plu à déconsidérer » (Branco, 2019).

Phạm Quỳnh fait figurer la chanson dans l'annexe d'un exposé qu'il adressait en 1930 à la Société de géographie. Sous la prudence du propos, résonne un message adressé à l'occupant colonial. On peut faire accepter beaucoup au paysan, mais il ne faut pas pour autant l'imaginer insensible au mépris. Le pouvoir colonial est resté sourd à tous les messages de cet ordre, dont ceux des membres (vietnamiens comme français) de l'École française d'Extrême-Orient. Toutes ses attentions allaient aux sociétés cotées en Bourse qui exploitaient l'Indochine pour fournir charbon et autres matières premières... au Japon impérial en marche vers la guerre de conquête. Dorgelès visite les Charbonnages du Tonkin,

[société] formidablement riche : les 74000 actions qui représentaient à l'émission 16 millions valent plus d'un demi milliard! Et savez vous combien ce royaume du charbon rapporte à l'Indochine, à la France ? Rien. Je dis rien, car je ne vais pas compter les quelques francs de taxe superficielle, les quelques sous de taxe minière. Il en est des Charbonnages comme de la plupart des riches entreprises de là-bas : de puissants inconnus se partagent les bénéfices, sucent la moelle de ce pays. Ni argent ni charbon : [la mine de] Hongay ne nous rapporte que la haine des milliers de coolies ${ }^{14}$.

Dorgelès lisait sur le visage des mineurs que la colonie ne continuerait pas plus de trente ans. L'Histoire a donné raison à son estimation. De là à ce que l'on apprenne à mieux lire ce que nous disent les visages de « ceux qui ne sont rien »... cela supposerait une écoute dont les conditions, manifestement, restent à réunir.

\section{Pour conclure}

Dans la situation délicate où se trouvent actuellement nos sociétés, on voit tel poète accoler écologie et poétique ${ }^{15}$ pour rebâtir, contre tout espoir, une espérance. On voit tels scientifiques recourir à la satire pour redire un message qui n'est pas entendu depuis des décennies ${ }^{16}$. De nouvelles oralités contestataires s'abritent, pour parler vrai, derrière la fragile protection qu'offre un registre satirique

\section{DorgeLÈs, 1925.}

15. Deguy, 2017.

16. Chapron et al., 2018. 
affiché ${ }^{17}$. L'étude de l'oralité, de la vive voix ${ }^{18}$ dans son épaisseur linguistique, sociale, et psychologique nous rapproche d'eux (espérons-nous) en nous plaçant à l'écoute des gens.

\section{Bibliographie}

Branco Juan, 2019, Crépuscule, Au diable vauvert, Paris, 320 p.

Chamberlain James, 1990, “Thao Hung or Cheuang: A Tai Epic Poem” in Mon-Khmer Studies, n ${ }^{\circ}$ 18-19, pp. 14-34.

Chapron Guillaume, Levrel Harold, Meinard Yves \& Courchamp Franck, 2018, "A Final Warning to Planet Earth" in Trends in ecology \& evolution, $n^{\circ} 33 / 9$, pp. 651-652.

Deguy Michel, 2017, L'Envergure des comparses : écologie et poétique, Hermann (coll. Le Bel Aujourd'hui), Paris, 176 p.

Dorgelès Roland, 1925, Sur la route mandarine, Albin Michel, Paris, 317 p.

Ferlu Michel, 1979, « Le récit khamou de Chuang et ses implications historiques pour le nord-Laos » in Asie du Sud-Est et monde Insulindien, $\mathrm{n}^{\circ} 10(2-4)$, p. 327-365.

Fónagy Ivan, 1983, La Vive voix : essais de psycho-phonétique, Payot (coll. Langages et Sociétés), Paris, 346 p.

Mathieu Christine, 2003, A History and Anthropological Study of the Ancient Kingdoms of the Sino-Tibetan Borderland-Naxi and Mosuo, Edwin Mellen Pr (coll. Mellen Studies in Anthropology, 11), Lewiston, New York, 496 p.

17. S'il ne fallait qu'un exemple, on retiendrait cette courte vidéo satirique (en anglais) : https://www.thejuicemedia.com/honest-government-ad-the-fires/.

18. FónAGY, 1983. 
CAHIERS DE LITTÉRATURE ORALE

154 Oralités contestataires - HORS-SÉRIE

Phàm Quỳnh, 1930, Le Paysan tonkinois à travers le parler populaire suivi d'un choix de chansons populaires, Đông Kinh ấn quán, Hanoi, réimpression en 1985 par Sudestasie, Paris. 126 p.

Plenel Edwy, 2019, La Victoire des vaincus. À propos des gilets jaunes, Paris, La Découverte, $187 \mathrm{p}$. 